\title{
André Malraux et Taiwan : le mythe moderne d'une communauté culturelle globale
}

André Malraux in Taiwan: The myth of a cultural global community

Stéphanie Shuling TSAI

\section{(2) OpenEdition}

Journals

Édition électronique

URL : http://journals.openedition.org/transtexts/201

DOI : 10.4000/transtexts.201

ISSN : 2105-2549

Éditeur

Gregory B. Lee

Édition imprimée

Date de publication : 1 mai 2006

Pagination : 182-192

ISSN : 1771-2084

\section{Référence électronique}

Stéphanie Shuling TSAI, « André Malraux et Taiwan : le mythe moderne d'une communauté culturelle globale », Transtext(e)s Transcultures 跨文本跨文化 [En ligne], 1 | 2006, mis en ligne le 13 septembre 2009, consulté le 19 avril 2019. URL : http://journals.openedition.org/transtexts/201 ; DOI : 10.4000/ transtexts.201

(ㄷ) Tous droits réservés 


\title{
André Malraux et Taiwan : le mythe moderne d'une communauté culturelle globale by Stéphanie Tsai Shuling
}

\begin{abstract}
Pour André Malraux, un homme se doit de tout posséder, jusque et y compris sa propre mort. Sa conception d'un musée imaginaire est dans le droit fil d'une telle attitude philosophique ; elle fait de la construction de l'homme par l'art un mythe «moderne » mais, aujourd'hui, au temps des mutations liées à la mondialisation, ce mythe tel qu'il a été élaboré demeure problématique. En effet, on peut se demander à quel point cette notion d'une communauté culturelle globale, site de conservation et espace synergique qui se prête à fabriquer les connaissances, les codes et les rituels, bref l'éducation, la formation et la culture du « bon goût » des citoyens, est pertinente à présent. A partir de trois articles consacrés à Malraux par des spécialistes taiwanais, nous pensons pouvoir questionner ce mythe d'une communauté globale, à la lumière des écrits d'un contemporain d'André Malraux, Maurice Blanchot, sur la mort.
\end{abstract}

\begin{abstract}
Andre Malraux admired in a man a demiurge capable of creating his own universe by fighting against fate, even against his own death. His concept of «imaginary museum » resonates alongside his metaphysical speculation: the will to engage oneself in the quest for the aesthetic, which, through art, leads to the construction of man. Facing the challenging mutation of globalization, the modern myth of « man by art » such as Malraux had elaborated remains problematic. In fact, a question might here be asked: to which point the notion of a cultural global community, a site of conservation, a synthetic space which lends itself to educate the good taste of citizens, could still be pertinent today ? Based on three articles consecrated to Malraux by Taiwanese specialists, this study intents to inquire into the myth of a global community in the light of the writings of a contemporary of Andre Malraux, Maurice Blanchot on the notion of death.
\end{abstract}

\begin{abstract}
对马尔侯(Malraux)而言, 人的价值就在于对命运的掌握, 全盘掌握, 甚至包括自己 的死亡。他有关 “想象的博物馆” 的观念是这样一种哲学态度的延伸:一个藉 由“艺术造人”完成的现代神话。在全球化风起云涌的今日，马尔候的神话特 别值得省思。实际上，我们可以自问：在何种意义上这一全球文化共同体的观 念在当今才是贴切中肯的? 这一共同体是一种旨在制造知识、符码与仪式，短 期教育，培训与公民“高品味” 文化的预留领地与协同空间。从台湾学者有关 马尔侯的三篇文章出发，在同代人马尔候与白朗修 (Blanchot) 有关死亡的文 本的启示下，我们将期望能够对这一全球共同体的神话进行探讨。
\end{abstract}




\section{Malraux \\ malentendu à Taiwan}

L'œuvre d'André Malraux n'a pas rencontré à Taiwan un accueil aussi chaleureux que celui de Jean-Paul Sartre, son contemporain. D'après une enquête sur la réception de la littérature française à Taiwan pendant les 50 dernières années (de 1950-2001), nous n'avons compté que 10 articles consacrés à Malraux, y compris un mémoire de maîtrise. 1 Cette occultation masque peut-être un déni politique et idéologique, mais ce n'est pas notre intention d'en analyser la cause. Ce qui nous intéresse dans les articles publiés à Taiwan, c'est la révélation de deux images plutôt opposées de Malraux : d'un côté, un humaniste qui a réussi à esquisser la description d'une condition humaine qui met en valeur la grandeur universelle de l'homme; de l'autre, un révolutionnaire qui tente, par sa conception de l'art, de mettre toutes les civilisations humaines dans le même cadre sans prendre en considération les contextes socio-historiques.

Dans son article intitulé «Révolutionnaire et romancier amateur : le grand écrivain du XXe siècle André Malraux», Chin Henh-Jei explique pourquoi Malraux a été mal reçu à Taiwan : D'abord Malraux est difficile à traduire en chinois en raison des complexités de ses phrases et des émotions enchevêtrées qu'elles recèlent. Ensuite, la réflexion métaphysique---surtout la notion de la mort s'éloigne trop de la pensée chinoise. Enfin, l'on entend la voix de Malraux derrière tous ses héros romanesques; les réactions, les perspectives et même la manière d'éprouver l'horreur sont toutes d'un mode « occidental,» ou plus précisément, «malrucien ». D'après Chin, le souci de Malraux porte sur l'archétype de l'Homme dans la tradition européenne dont la valeur est ancrée dans la civilisation greco-romaine et le christianisme, ce qui rend difficile aux lecteurs chinois d'aborder l'œuvre de Malraux. Pourtant, à l'autre pôle de la réception, Malraux a reçu, malgré les différences culturelles, des marques de respect dans d'autres articles tels que celui de Chen Chien-Hong, qui le reconnaît comme grand écrivain-penseur humaniste dont la pensée s'aligne avec de grands noms philosophiques - de Pascal à Nietzsche. ${ }^{3}$

A part ces deux opinons opposées, nous voudrions signaler encore deux articles qui représentent, pourrions-nous dire, la troisième image de Malraux reçue par détour des critiques américains à travers la traduction anglaise de ses œuvres. Chang Wan-Cheng en étudiant le mot «musée » entendu par Malraux dans son Musée imaginaire, a indiqué que le terme d'« imaginaire » a une acception différente dans la version anglaise. ${ }^{4}$ D'après la critique américaine, Rosalind E. Krauss, le titre traduit «Museum without Walls » dénote

1 L'enquête fut récemment menée par le département de français de l'Université Tamkang. 2 金恒杰Chin Henh-Jei, «Kechuan gemingjia piaoyou xiaoshuojia : Ershishiji weida de zuojia Angderui Maerhou »客串革命家票友小說 家: 二十世紀偉大的作家昂德瑞馬爾侯[Révolutionnaire et romancier amateur : le grand écrivain du XXe siècle André Malraux], Dangdai, 1988 (28), 8, p.76, 3 陳健宏 Chen Chien-Hong, «Yishu yu mingyun : Maerhou de renbenzhuyi yishuguan »藝術與命運: 馬爾侯的人本主義藝術觀 [Art et destin : La dimension artistique humaniste d'André Malraux], Dangdai, 1989 (40), 8. 4 張婉真 Chang Wan-Cheng, «Andelie Maerhou de wuqiang meishuguan 》安德烈馬爾侯的無牆美術館 [Le musée sans mur d'André Malraux], Lishi WenWu, 2001 (99), 10 ; André Malraux, The Voice of Silence, reissued 1978 by Princeton University Press (part I: Museum without 
un écart linguistique et culturel à l'égard de la pensée de Malraux : «imaginaire » chez Malraux est plutôt liée à une activité intellectuelle qui évoque la puissance de l'imagination et du jugement esthétique, tandis que «without walls » connote plutôt le sens de «sans frontière » et renforce ainsi la vision d'une image concrète préférée des Anglo-Saxons. ${ }^{5}$ ॥ est intéressant de noter que Madame Chang a elle-même adopté la version anglaise pour le titre de son article : «Maerhou de wuqiang meishuguan» (Musée sans mur d'André Malraux.) Sans entrer ici dans le détail des différences entre meishuguan et bowuguan en chinois (le premier signifie «musée des beaux-arts », pas forcément ancien, et le second, «musée d'art ancien »), nous nous demandons si la réception d'André Malraux à Taiwan n'a pas consisté à métamorphoser ou transformer le terme d'« art » ou de « musée » en leur donnant la valeur d'un signe. L'article de Chou Yuan-Hung essaie justement d'interpréter les idées de Malraux comme un « discours » portant sur la métamorphose du Bouddha grec et la mythopoièse du musée et de l'histoire d'art. - $^{-}$

L'image contestée de Malraux aurait dû mériter à Taiwan des études plus étendues, plus approfondies et bien sûr, plus polémiques. Nous allons retourner plus tard aux articles déjà cités au cours de notre intervention. Personnellement, à part La condition humaine que j'ai lu il y a plus de 15 ans, ce qui m'a amené à relire Malraux, c'est un petit livre intitulé Lazare (1974), un récit autobiographique sur l'expérience de "frôler la mort », qui m'a fait penser à un petit récit L'instant de ma mort (1994) de Maurice Blanchot, l'auteur pivot de mes recherches depuis ma thèse de doctorat. ${ }^{7}$ Je partage l'avis de Chin Henh-jei qui a insisté sur l'importance de bien saisir les valeurs européennes pour aborder les textes de Malraux, mais d'un autre point de vue. Ce qui est remis en question dans ma lecture de Malraux, c'est justement ce en quoi consiste ces valeurs ou plus précisément, comment elles se fabriquent comme « mythe » ou « discours » dans une époque et une société données.

Beaucoup d'études ont été consacrées au débat intellectuel entre Sartre et Blanchot, après la guerre, sur la conception de l'engagement. Sartre postule le concept de légitimité de la responsabilité des intellectuels, alors que pour Blanchot, l' "être-intellectuel » en tant que « représentant de l'universel » ou « la conscience de tous » n'a aucun statut pertinent ou permanent. $\stackrel{-}{ }$ André Malraux, pourrait-on dire, partage l'idée de l'engagement en s'efforçant de diffuser l'art à un plus large public par les musées imaginaires. L'homme « moderne » est censé être capable d'accéder à un monde de l'art autrefois réservé à une élite. Benoit Denis a ainsi écrit dans Littérature et engagement:

\footnotetext{
5 Rosalind E. Krauss, "Postmodernisme's Museum Without Walls" in Reesa Greenberg (and al.), Thinking about Exhibitions, Routledge, 1999, pp. 341-342, 6 Chou Yuan-Hung, "Greek Buddha : On the Journey of an Icon and the Mythopoesis of Museum and Art History", www.cc.nctu. edu.tw 7 André Malraux, Oeuvres complètes III, Pléiade, Gallimard, 1996. pp. 787-879. 8 «Qu'est-ce que la littérature ?» (1947) de Sartre est souvent opposé à «Littérature et le droit à la mort» de Blanchot. Phillips Watts a écrit: "Blanchot was the single most important critic for readers searching for an antidote to existential models of literary criticism: theories of textuality and vertiginous undecidability in an attempt to get beyond the calls for responsibility and judgment", Allegories of the Purge: How Literature Responded to the Postwar Trials of Writers and Intellectuals in France, Stanford University Press, 1998. Paul de Man parle d" "Impersonality in Blanchot" (1966): "If Blanchot's work moves us beyond judgment, the keystone of Sartrean literary analysis, it is precisely because his writing dissolves the subject, author and reader. Dans Littérature et engagement, Benoit Denis écrit: "C'est au cours des mêmes annés que l'on commence à prendre vraiment la mesure d'un fait dont la génération sartrienne n'avait pas perçu l'importance fondamentale : celui du génocide et des camps d'extermination. ..Mais c'est seulement au cours des années cinquante que cette problématique majeure fait véritablement sentir ses effets et amène des écrivains, tels Blanchot ou Duras (à la suite de Bataille), à placer au coeur de leur projet d' écriture la question de l'indicible et du silence : à nouveau, c'est ici à positivité de l'engagement sartrien qui se trouve mise en cause, ainsi que sa conception quasi instrumentale du langage. », Littérature et engagement, Gallimard, 2000, pp. 282-283.
} 
La littérature engagée, telle que Malraux la pratique, transcende les faits et l'expérience pour les doter d'une signification universelle----la révolution n'y est rien d'autre que l'avatar contemporain de l'éternel affrontement tragique de l'homme au destin et à l'Histoire. ${ }^{9}$

Un demi-siècle après leurs débats sur la valeur et le monde, aujourd'hui au temps des mutations liées à la mondialisation, l'on peut se demander à quel point la notion d'une communauté culturelle globale est pertinente à présent.

En ce qui concerne la mise en relief du travail Blanchot-Malraux, je me permets de signaler d'abord un essai d'Henri Godard, intitulé "L'expérience existentielle de l'art ", écrit à l'occasion de la récente publication de Malraux: Ecrits sur l'art à la Pléiade..$^{10}$ Dans cet essai, Henri Godard a consacré un chapitre au texte de Blanchot sur Malraux (1950) intégré plus tard au recueil intitulé Amitié (1971)..11

Par la constance et la radicalité de sa référence à la mort, à l'absence, au néant, [la pensée] de Blanchot est un pôle de notre réflexion sur l'art, dont Malraux pourrait bien incarner l'autre. 12

Plus tard Godard affirme dans un entretien que "Blanchot est à l'opposé de Malraux; II voit dans l'œuvre la révélation suprême d'une négativité essentielle $» . .13^{13}$ Avant de marquer la divergence entre les deux pensées sur le "réel ", Godard a mis l'accent plutôt sur l'accord et « l'étonnante consonance » des deux écrivains. »14 En prenant la position du côté de l' " admiration et de la confiance, non de la suspicion et de la défiance » à l'égard des interrogations d'ordre métaphysique sur l'art de Malraux, Godard propose de recourir aux expériences "actuelles » de chaque personne pour savoir " pourquoi, dans une existence, l'art peut, selon sa formule, devenir 'nécessaire' ? ». $\frac{15}{5}$

Nous allons voir que la distinction entre Malraux et Blanchot sur la notion de la mort est évidente. Ce qui nous intéresse, c'est de transporter la structuration du texte de départ dans un autre réseau de connotations, à l'intention de lecteurs dont la culture maternelle ne s'inscrit pas dans la lignée greco-latine. Le problème de la réception de Malraux à Taiwan ne réside pas dans la compréhension exacte du message transmis par Malraux, mais l'enregistrement des traces de lectures et la définition des limites de signification. II ne s'agit pas d'argumenter sur le droit de tel ou tel de parler au nom de la Chine ou de l'Art, mais de se poser la question autrement : comment le discours de Malraux construit le «mythe » de la vie à partir de la mort ? Et comment le mythe aide à formuler l'idée de la « communauté » et tous les codes de signification qui s'y enchaînent ? II nous semble que la notion de la mort propre à ces deux écrivains est la clé permettant d'entrer au coeur de leur discours.

9 |bid, p. 254. L'entrée de Sartre en littérature est plutôt tardive, datée de l'extrême fin de l'entre-deux-guerres. D'après Benoit Denis, De Beauvoir et Sartre « lisaient Malraux avec un mélange significatif d'admiration et d'irritation » comme ils connaissaient bien les milieux littéraires qui gravitaient autour du PCF, grâce à leur ami Paul Nizan, $p$ 266, 10 André Malraux, Ecrits sur l'art I.II., La Pléiade, Gallimard, 2004. 11 "Le musée, I'art et le temps ", "Le mal du musée. », dans Maurice Blanchot, Amitié, Gallimard,1971, pp. 21-61. 12 Henri Godard, L'expérience existentielle de l'art, Gallimard, 2004, p. 107. 13 L'interview a été accordée par Jérôme Serri, Lire, novembre, 2004. 14 Godard, L'expérience existentielle de l'art, p.113, 15 Ibid, p.16. 


\title{
Fabrication de mythes à partir de la mort
}

\begin{abstract}
Lazare, une oeuvre «testamentaire » écrite par le narrateur-auteur (Je-Malraux) au sortir d'une maladie du sommeil, se constitue de deux parties inter lacées : un récit des événements imprévisibles et bouleversants déjà traité il y a trente ans dans Les Noyers de l'Altenburg : la première attaque allemande par les gaz à Blogako sur la Vistule, en 1916 dans l'autre partie, le Je-Malraux décrit son hospitalisation, ses jours de malade traversés des souvenirs de sa vie antérieure ${ }^{16}$ Comme Marius-François Guyard l'a bien indiqué dans l'introduction, le titre Lazare suggère la résurrection de l'écrivain, et la présence de la mort dans le corps du malade gouverne le livre de la première à la dernière ligne. " Lazare est comme la synthèse fébrile de toute une vie, de toute une oeuvre " qui cherche à trouver l'apaisement à l'approche de la mort.

En face de la mort qu'il a cru pouvoir « posséder » par l'action, le sentiment qu'il éprouve le plus souvent dans l'hôpital de la Salpêtrière, c'est la stupéfaction. Le fait qu'il ne souffre pas le mène à penser la mort dans un état ignoré :
\end{abstract}

Ce qui s'est passé n'a rien de commun avec ce que j'appelais mourir.

A l'instant de basculer (j'avais quitté terre) j'ai senti la mort s'éloigner ; pénétré, envahi, possédé, comme par une ironie inexplicablement réconciliée, qui fixait au passage la face usée de la Mort...17

Le sentiment éprouvé envers la mort pourrait être différent, mais « la vue de l'homme ", écrit Malraux, " a peu changé depuis trente ans »..18 Le héros malrucien sort toujours conquérant du déploiement d'un spectacle sublime mettant en scène sa grandeur contre sa misère. En face de la mort, Malraux parle toujours d'un ton dominateur :

La métamorphose en conscience, de l'ignorance de la mort, ou la métamorphose de toute connaissance en croyance, n'est-elle pas semblable aux épiphanies ? Mon errance hors de la terre pour rapporter les comprimés est aussi une épiphanie des ténèbres. La révélation est que rien ne peut être révélé [...] L'inconcevable n'a aucun attribut - pas même la menace : I'homme ne devient pas plus scorpion que damné, et pas plus néant que scorpion..$^{19}$

16 André Malraux, Oeuvres Complètes II, La Pléiade, Gallimard, 1996, p. 787. 17 André Malraux, Oeuvres Complètes III, La Pléiade, Gallimard, 1996, p. 879, 18 |bid, p. 817, 19 |bid, pp. 877-878. 
Malraux ne croit pas à la Rédemption, ni à la foi chrétienne. A travers la mémoire des scènes confondues de vie et d'œuvre, l'écrivain retourne à la terre à l'aide d'un terme triomphant : « fraternité, que le destin n'efface pas ». Malraux écrit dans Lazare «... je cherche la région cruciale de l'âme où le Mal absolu s'oppose à la fraternité. »20 La rencontre de la Mort incarnée du Mal absolu est l'événement même qui évoque en soi la trouble et la puissante action des " grands mythes de la révolte depuis Antigone ». ${ }^{21}$ Marc Bertrand a ainsi commenté sur le goût du mythe chez Malraux :

Voici l'homme obligé de se réaliser contre plus fort que lui. Nous avons toujours affaire à un mythe, et le réel continue de subir la loi de l'irréel tout puissant.... En fait, ce sur quoi Malraux met l'accent, c'est sur la priorité étonnante et inexplicable de l'irréel littéraire qui semble parfois précéder le réel historique et le plier à sa loi. $\underline{22}$

Marc Bertrand en nommant Malraux « mythomaniaque » l'accuse de

se livrer à une véritable entreprise de subversion, à un abus de l'affirmation et de l'invention de soi par soi, dans une solitude orgueilleuse, par-delà le bien et le mal pour parler comme Nietzsche. $\underline{23}$

Marc Bertrand propose ainsi de remplacer la «mythomanie» malrucienne par la "mythopoièse » telle que conçue par J.F Lyotard. Le rêve mythomaniaque de Malraux est celui de l'individu qui cherche à se donner visage, c'est une invitation à se réaliser, soit dans l'action, soit dans la création ; tandis que la «mythopoièse » signale le processus de la fabrication de mythes.

L'on ne peut pas s'empêcher ici de se poser une question : si c'est la mythomanie malrucienne qui empêche les lecteurs chinois d'entrer dans le monde de Malraux à cause de l'écart linguistique, social et philosophique, le recours à la mythopoièse nous aiderait-il à mieux cerner le problème des valeurs européennes, de la métamorphose de l'art aussi bien que la notion d'une communauté culturelle et universelle?

II me semble que la distinction entre la «mythomanie » et la "mythopoièse » réside dans la distance que l'on prend à l'égard de la mort et ainsi de la réalité. Malraux écrit dans $L a$ voie royale : "Tout aventurier est né d'un mythomane », ce qui est commenté ainsi par Marc Bertrand : "La mythomanie est pour Malraux beaucoup plus qu'un cas pathologique ...car elle consiste moins à fuir sa réalité, qu'à vouloir se la donner. „24 La création de la réalité dépend de la croyance de pouvoir posséder la mort. La mythomanie mélange ainsi la vie et la création, la fiction et la réalité, tout comme Lazare nous l'a bien montré. Pourtant, ce qui nous met mal à l'aise, ce n'est pas la frontière brouillée entre le réel et l'irréel, c'est plutôt cette propension à proposer cette mythomanie comme Vérité, à partir de laquelle Malraux prône l'établissement d'une communauté universelle.

Dans L'instant de ma mort de Maurice Blanchot tout comme dans Lazare, le narrateur raconte

20 Malraux, Oeuvres Complètes II, p. 788, 21 lbid, p. 788, 22 Marc Bertrand, « André Malraux : du mythomane au postmoderne ", http://www. andremalraux.com/malraux/articles, 23 bid. Voir aussi Olivier Todd, André Malraux une vie, Paris, Gallimard, 2001. 24 Bertrand, "André Malraux : du mythomane au postmoderne ». 
l'expérience de la mort d'un «jeune homme " qui échappe à peine au fusil des Allemands pendant la guerre. $.25 \mathrm{~A}$ la rencontre de la mort, l'auteur narrateur qui se dit «il » éprouve un sentiment de «légèreté extraordinaire », " une sorte de béatitude » et " une allégresse souveraine ": nous pouvons distinguer tout de suite un ton différent de celui de Malraux :

A sa place, je ne chercherais pas à analyser ce sentiment de légèreté. II était peut-être tout à coup invincible. Mort - immortel. Peut-être l'extase. Plutôt le sentiment de compassion pour l'humanité souffrante, le bonheur de n'être pas immortel ni éternel. Désormais, il fut lié à la mort, par une amitié subreptice..$\underline{6}$

1
8
8

La mise en relief du travail de Blanchot et de Malraux se justifie plus loin par une anecdote qui termine L'instant de ma mort:

Plus tard, revenu à Paris, il rencontra Malraux. Celui-ci lui raconta qu'il avait été fait prisonnier (sans être reconnu), qu'il avait réussi à s'échapper, tout en perdant un manuscrit... Qu'importe ! Seul demeure le sentiment de légèreté qui est la mort même ou, pour le dire plus précisément, l'instant de ma mort désormais toujours en instance. ${ }^{27}$

A la rencontre de la mort, Malraux recourt à la fraternité pour lutter contre la fin de la vie, tandis que Blanchot éprouve presque une amitié étrange à l' égard de la mort même. Pour Blanchot, citant Rilke (les " Sonnets à Orphée »), ce que l'artiste ou le poète cherche à entendre, c'est une invitation : "Sois toujours mort en Eurydice, afin d'être vivant en Orphée». $2 \underline{2}$ Cette invitation qui implique une duplicité étant l'essence non essentielle de l'œuvre d'art:

au fond de cet ordre, toujours mort a pour écho toujours vivant, et vivant ne signifie plus ici la vie, mais, sous les couleurs de l'ambiguité rassurante, signifie la perte du pouvoir de mourir. $\underline{29}$

La perte de la mort est interprétée comme perte de pouvoir et possibilité. Blanchot propose de lire le mot «mort » sans négation, de lui retirer le tranchant de la décision et le pouvoir de nier. L'homme ne s'adresse jamais à la hauteur de l'Impensable qui est tout à fait hors la portée de l'intelligence humaine. La mort étant écho de l'Impensable est un événement qui se livre à l'indistinct et à l'indéterminé. Pour Blanchot, la littérature ou l'art n'est ainsi jamais possibilité d'imposer une signification fixe à la mort ou à la vie. L'art comme image, mot ou rythme, à son extrémité, n'est qu'une forme de substance matérielle qui rend invisible I'Impensable dans son invisibilité ; toute forme d'art étant une négation qui ne peut pas s'empêcher de se nier perpétuellement dans le mouvement de la négativité.

25 Publié en 1994 par Fata Morgana et relié dans le même livre avec Jacques Derrida Demeure: Maurice Blanchot, Standford University Press, 2000, 26 bid, p. 4, 27 bid, p. 10, 28 Maurice Blanchot, L'Espace littéraire, Gallimard, 1955, p. 324. 29 Ibid, p. 324. 


\section{Parole, vérité et communauté}

Dans Le livre à venir, chapitre V «La douleur du dialogue », Blanchot écrit sur Malrauxromancier. Pour Blanchot, Malraux a rendu art et vie à une attitude très ancienne, datée de Socrate, qui croit à l'efficacité de la parole. Par la discussion, l'homme est censé capable d'arriver à l'aptitude d'une « vraie vie » dont la cohérence ne doit se fonder que sur la parole raisonnable. Grâce à la discussion sur la vie aussi bien que sur la réconciliation de l'art et de la politique, une communauté se met à prendre forme et une vérité commune à tous se dessine :

Ces simples dieux humains, un instant au repos sur leur humble Parnasse, ne s'injurient pas, ne dialoguent pas non plus, mais discutent, car ils veulent avoir raison, et cette raison est servie par la vivacité flamboyante des mots, lesquels toutefois restent toujours en contact avec une pensée commune à tous et dont chacun respecte la communauté préservée.

D'après Blanchot, la constitution d'une communauté des dieux-humains est permise seulement dans la reconnaissance de l'efficacité de la parole fondée sur la soi-disant « intelligence ». Cette "intelligence », différente de la « raison », est en effet, une passion qui

s'intéresse à tout : les mondes, les arts, les civilisations, les débris de civilisations, les ébauches et les accomplissements, tout lui importe et tout lui appartient. Elle est l'intérêt universel qui comprend tout passionnément, tout par rapport à tout. .13

La notion de la mort chez Blanchot diffère ainsi de celle de Malraux justement par la certitude du pouvoir de la parole. La parole, malgré la prolifération des formes de discussion, ne mène ni à la révélation, ni à la possession de la soi-disant « vérité ", qui se prête à solidifier une communauté telle que Malraux l'a affirmée dans ses écrits. Au contraire, la "parole » authentique pour Blanchot, c'est la parole incessante de l' " Impersonnel » qui échappe à la portée de l'intelligence humaine. Ce que Blanchot recherche, c'est un espace vidé par le départ des dieux, un espace qui se métamorphose en tant que parole incessante. Contrairement à l'attitude apparemment «engagée " de Malraux, Blanchot propose une approche plutôt « désœuvrée » de la parole :

Ecrire sans « écriture ", amener la littérature à ce point d'absence où elle disparaît, où nous n'avons plus à redouter ses secrets qui sont des mensonges, c'est la «le degré zéro de l'écriture ", la neutralité que tout écrivain recherche délibérément ou à son insu et qui conduit quelques-uns au silence.[...] Le langage devenu la profondeur désœuvrée de l'être, le milieu où le nom se fait être, mais ne signifie ni ne dévoile.

30 Maurice Blanchot, Le livre à venir, Gallimard, 1959, p. 210, 31 bid, p. 210, 32 bid, pp. 282-283. 
En répondant à La communauté désoeuvrée de Jean-Luc Nancy, Blanchot met en cause, dans La communauté inavouable (1983), la notion même de «communauté » qui porte sur la croyance de «commun»:

...commun à ceux qui prétendraient appartenir à un ensemble, à un groupe, à un conseil, à un collectif, fut-ce en se défendant d'en faire partie, sous quelque forme que ce soit.. .3

« Communauté » en tant qu' " œuvre » suppose le principe d'une humanité « immanente » qui égalise tout jusqu'à la notion d' «origine», ce qui est, aux yeux de Blanchot, « le totalitarisme le plus malsain. „34 $^{34}$ Blanchot voit dans cette exigence d'une immanence absolue un individu indéfiniment répété :

ainsi mortel et immortel : mortel dans son impossibilité de se perpétuer sans s'aliéner, immortel, puisque son individualité est la vie immanente qui n'a pas en elle-même de terme. $\frac{35}{5}$

Ce qui est intéressant pour nous, c'est de distinguer dans ce discours de Blanchot l'idée de " reproduction » de l'individualité immanente qui caractériserait la communauté culturelle et artistique dans Le Musée imaginaire de Malraux.

Pour Blanchot, la communauté est «inavouable » comme la présupposée réciprocité ou amitié de l'homme à l'homme (le rapport du Même avec le Même) n'est qu'une illusion. Si l'on introduit l'Autre comme irréductible au système du Même, on aurait toute une autre relation «dissymétrique " qui demande un différent mode éthique, par conséquent, la communauté serait condamnée à " désoeuvrer ». Pourtant, on aurait tord de penser que Blanchot tend à nier tout simplement l'exigence de communauté ; ce qui est mis en jeu, c'est le paradoxe qu'introduit ici l'adjectif possessif «mon » : dans l'exigence d'un groupe d'amitié et de fraternité auquel «je » cherche l'appropriation, «ma mort » que personne ne puisse partager risque de ruiner toute appartenance.

A partir de la mort, du principe d'incomplétude, et d'insuffisance, Blanchot propose une autre possibilité de "communauté ", un lien qui résulte du manque et qui ne se fonde pas dans l'objectif de former une substance d'intégrité :

L'être, insuffisant, ne cherche pas à s'associer à un autre pour former une substance d'intégrité. La conscience de l'insuffisance vient de sa propre mise en question, laquelle a besoin de l'autre ou d'un autre pour être effectuée. .36

Non pas reconnu, mais contesté ou même nié par les autres, c'est dans cette privation que l'individu se rend conscient (la contestation ou la négation des autres étant l'origine de sa propre conscience). Nous pouvons affirmer ici la notion de subjectivité blanchotienne : exister, l'être conscient de

l'impossibilité d'être lui-même, d'insister comme ipse, s'éprouvant comme extériorité toujours préalabe, ou comme existence de part en part éclatée, ne se composant que comme de décomposant constamment, violemment et silencieusement. ${ }^{37}$

33 Maurice Blanchot, La communauté inavouable, Minuit, 1983, pp. 9-10 34 |bid, p. 11. 35 |bid, p. 12.36 |bid, p. 16.37 Ibid, p. 16. 
Malraux rêve d'une communauté universelle qui se fonde sur la parole raisonnable réconciliant l'art et la politique. Pourtant, d'après Blanchot, cette communauté « des dieuxhuminas » telle qu'elle a été esquissée chez Malraux, loin d'être une manifestation qui relie toute forme différente artistique et culturelle, se détache encore plus loin de la notion de «l'unité de l'origine ».

\title{
Métamorphose en double versions
}

Dans l'article "Le musée, l'art et le temps », Blanchot remet en question l'idée du musée imaginaire de Malraux. $\frac{38}{-}$ La valeur absolue de l'art, au niveau esthétique aussi bien qu'éthique telle que Blanchot l'affirme est une sorte d'art qui se ferme sur lui-même, d'avoir en soi son excellence et sa signification. Par sa distance à l'égard du monde, l'art se prête comme espace où le monde se remet sans cesse en question. En refusant toute interprétation déterminée, l'art assume la vérité de la non-vérité, puisque " toute la perspective dans laquelle le musée, le monde et l'artiste nous étaient apparus change. ${ }^{39}$ Le Musée étant l'univers propre à l'art et à la liberté de l'histoire, éclaircit l'idée de métamorphose par excellence. Le Musée en tant que lieu de métamorphose, loin d'être une manifestation métaphysique ou une illustration de la culture, se détache encore plus radicalement de la notion "du devoir, de l'obligation purement morale, de sauver la civilisation et de préserver l'homme " telle qu'elle a été élaborée dans le musée imaginaire de Malraux. Pour Blanchot, si l'on cherche une "unité " qui relie toute forme différente de l'art, c'est un langage spécifique qui retourne sans cesse à « l'origine » de l'art qui désoeuvre toute tentation d'imposer une signification. En parlant de Proust, Blanchot explique ce qu'il entend par la métamorphose de l' « origine »:

\begin{abstract}
Eprouver à l'état pur, en éprouvant la transformation du temps en espace imaginaire (l'espace propre aux images), en cette absence mouvante, sans événements qui la dissimulent, sans présence qui l'obstrue, en ce vide toujours en devenir: ce lointain et cette distance qui constituent le milieu et le principe des métamorphoses et de ce que Proust appelle métaphoriser.... 40
\end{abstract}

L'acte de créer est ainsi considéré comme une aventure, une migration ou une navigation ; c'est un vagabondage de l'artiste qui erre jusqu'au point fabuleux où il rencontre l'événement qui rend possible tout récit, au moment à partir duquel ce qui s'est passé devient réalité et vérité.

Ce qui diffère radicalement l'idée de Malraux et celle de Blanchot, c'est bien «la gloire de l'homme » qui cherche à se sauver, par l'art, de l'absurdité et de la contingence---donc de la menace de la mort. Pour Malraux, sans le travail de l'homme qui transforme le passé en un présent rayonnant et intelligible, nous trouverions dans le Musée rien d'autre que les ruines informées d'une durée sans mémoire, la pourriture du cadavre du temps :

Pour Malraux, la métamorphose est surtout la re-création de l'œuvre d'art par la reproduction

38 Maurice Blanchot, Amitié, Gallimard, 1971, p. 21.39 |bid, p. 37. 40 Blanchot, Le livre à venir, p. 23. 
Le musée impose une mise en question de chacune des expressions du monde qu'il rassemble, une interrogation sur ce qui les rassemble. Après tout, le musée est un des lieux qui donnent la plus haute idée de l'homme. Mais nos connaissances sont plus étendues que nos musées, ...Et le Musée Imaginaire poursuit sa métamorphose. ${ }^{41}$

retirée hors du contexte socio-historique, grâce à laquelle on pourrait former une communauté culturelle globale au niveau du discours :

\begin{abstract}
Et le musée supprime de presque tous les portraits (le fussent-ils d'un rêve), presque tous leurs modèles, en même temps qu'il arrache leur fonction aux oeuvres d'art : il ne connait plus ni palladium, ni saint, ni Christ, ni objet de vénération, de ressemblance, d'imagination, de décor, de possession; mais des images des choses, différentiels des choses mêmes, et tirant de cette différence spécifique leur raison d'être. II est une confrontation de métamorphoses. .2 $^{2}$
\end{abstract}

Cette idéalisation et cette éternisation de la fin n'est pourtant qu'une illusion pour Blanchot, une illusion qui se trahit par l'intention de cacher l'angoisse de l'étrangeté que l'homme éprouve face au temps. L'art comme image, est un "arrêt de mort ", une limite auprès de l'indéfini ou une interruption au sein du remuement, l'instant de l'absence devenue forme matérielle. Ce qui relie toute image artistique, c'est ce qui réside au cœur même de l'image - la mort comme recommencement, ressassement éternel. L'art comme image, est le négatif du néant, le négatif qui fait écho à ce qui ne peut jamais être nié.

Curieusement, l'idée de Blanchot qui remet sans cesse en question l'efficacité des paroles produites sur le beau et le vrai nous emmène paradoxalement à mieux apprécier le discours de la métamorphose malrucienne par le biais d'une autre perspective : dans le musée imaginaire, ce que Malraux a réussi à nous révéler, ce n'est pas l'authenticité ni la possession des objets d'art, mais le pouvoir de savoir créer des mythes, à partir d'eux.

Pour conclure, retournons aux essais mentionnés plus haut. En citant Jean Lacouture, Chin a vivement questionné la «mystification » des expériences « vécues » de Malraux en Chine, alors que pour Chou, l'authenticité du vécu n'est plus le gage du constat. ${ }^{43}$ Chou traite dès le début de son étude le discours de Malraux comme une démonstration de «mythopoièse.» Dans le cadre du post-colonialisme, Chou signale que le musée étant une institution du pouvoir, sert à façonner l'identité d'un Etat moderne dès le XIXe siècle en Europe ; site de conservation et espace synergique qui se prête à fabriquer les connaissances, les codes et les rituels, bref l'éducation, la formation et la culture du " bon goût » des citoyens. II s'agit de collectionner, catégoriser des objets d'art et d'inventer des systèmes de discours pour les remettre en cadrage "significatif ». Si l'on apprend à parler et à faire comme Malraux, ce qu'il nous a inspiré, ce n'est pas une question de l'authenticité de la représentation, mais le droit de reproduction et celui de fabriquer des discours de signification. Citons Malraux une dernière fois :

\footnotetext{
41 André Malraux, " Musée imaginaire », Ecrits sur l'art, I. Pléiade, Gallimard 2004, pp. 204-331. 42 Ibid, p. 204.
}

43 André Malraux, Une vie dans le siècle, Paris, Seuil, 1973, pp.112-113. 